\title{
Temtamy syndrome caused by a new $C 12$ orf57 variant in a Chinese boy, including pedigree analysis and literature review
}

\author{
YANQIN WANG ${ }^{1,2^{*}},{\text { MING } \mathrm{LI}^{3 *}, \text { YUANYUAN LUO }}^{1,2^{*}}$, XIN ZHAO ${ }^{1,4}$, \\ SHUANG LIAO $^{1,2}$, LI JIANG ${ }^{1,2}$, XIUJUAN LI ${ }^{1,2}$ and MIN ZHONG ${ }^{1,2,5}$ \\ ${ }^{1}$ Department of Neurology, Children's Hospital of Chongqing Medical University; ${ }^{2}$ Pediatric Research Institute, \\ China International Science and Technology Cooperation Base of Child Development and Critical Disorders, \\ National Clinical Research Center for Child Health and Disorders, Chongqing Engineering Research Center of \\ Stem Cell Therapy, Ministry of Education Key Laboratory of Child Development and Disorders, Chongqing 400014; \\ ${ }^{3}$ Department of Respiration, Kunming Children's Hospital, Kunming, Yunnan 650000; ${ }^{4}$ Huazhong University of Science \\ and Technology Union Shenzhen Hospital, Shenzhen, Guangdong 518052; ${ }^{5}$ Department of Pediatrics, \\ Qianjiang Central Hospital of Chongqing, Chongqing 409000, P.R. China
}

Received January 7, 2019; Accepted October 7, 2019

DOI: $10.3892 / \mathrm{etm} .2019 .8183$

\begin{abstract}
Temtamy syndrome is an extremely rare disorder caused by chromosome 12 open reading frame 57 (C12orf57) pathogenic variants. The present study reported a boy with Temtamy syndrome displaying global developmental delay, epilepsy and dysmorphic facial appearance. Whole-exome sequencing was performed to identify a novel homozygous pathogenic variant of C12orf57 (c.3G >C, p.Met1IIe), and the affected protein structure and function were predicted to be pathogenic. Additionally, clinical features of the other reported 56 patients with $C 12$ orf57 pathogenic variants were reviewed and compared. This study highlighted that C12orf57 pathogenic variants are mainly associated with global developmental delay, epilepsy and dysmorphic facial appearances. The clinical features were in accordance with the previously reported cases, except for those with recurrent infection, but without corpus callosum abnormalities. The present study reported the first Asian case to the best of our knowledge with Temtamy syndrome, and the novel C12orf57 pathogenic variant has not reported in any ethnic groups previously. The present study expanded the spectrum of C12orf57 pathogenic variants as well as the ethnic backgrounds of the affected patients.
\end{abstract}

Correspondence to: Dr Min Zhong, Department of Neurology, Children's Hospital of Chongqing Medical University, 136 Zhongshan, 2nd Road, Yuzhong, Chongqing 400014, P.R. China E-mail: zhongmin@hospital.cqmu.edu.cn

*Contributed equally

Key words: epilepsy, developmental delay, pathogenic variant, Temtamy syndrome, $\mathrm{C} 12$ open reading frame 57

\section{Introduction}

Temtamy syndrome (MIM no. 218340) is a rare disorder which was first described in 1991 and then published a full formal report in 1996, where three siblings affected appeared to have inherited an autosomal recessive gene $(1,2)$. It is characterized with mental retardation and multiple congenital anomaly, with symptoms including variable craniofacial dysmorphism, ocular coloboma, seizures and abnormalities in the corpus callosum and thalamus (1-3). Of note, the chromosome 12 open reading frame 57 (C12orf57) encodes a 126-amino acid cytoplasmic protein of unknown function was reported to be required for human corpus callosum development $(3,4)$. In 2013, pathogenic variants of C12orf57 were first reported to cause Temtamy syndrome $(3,4)$. At the present time, only seven pathogenic variants have been illustrated (1-6). Those of Middle Eastern descent are particularly susceptible to C12orf57 pathogenic variants, with 54/56 (96.4\%) of all the reported patients from Middle Eastern countries, predominantly Saudi Arabia (3-7).

The present study reported the first East Asian patient with global developmental delay and epilepsy caused by a novel homozygous C12orf57 pathogenic variant, and presented a brief review of all the previously published cases.

\section{Patients and methods}

Patients. Ethics approval for this study was obtained through the Institutional Review Board of Children's Hospital of Chongqing Medical University. Written informed consent was obtained from the parents of the patient for the publication of this case report and accompanying images.

The present case is of a boy born to first-cousin consanguineous Chinese parents of Hui nationality at 40 weeks and 5 days of gestation, gravida 2 para 2 . The boy was born by normal delivery with a birth weight of $3,510 \mathrm{~g}$, while the birth length and head circumference were unknown. His elder sister is 7 years old and healthy as of October 2019. 
The patient was admitted to the Neurology ward at the Children's Hospital of Chongqing Medical University (May 2018) at the age of 8 months and 28 days because of four tonic-clonic seizures in 10 days, as well as pneumonia which had lasted for 1 week. All of the available clinical characteristics of the patient, along with the aforementioned auxiliary examination results are summarized in the present study.

Whole-exome sequencing and bioinformatics analysis. Based on the hg19/GRCh37 reference, whole-exome sequencing was performed on the proband and the parents at Beijing Mygenostics Co., Ltd. Several online databases containing data from different ethnic groups were used as follows: Genome Aggregation Database (gnomAD, http://gnomad.broadinstitute.org/); 1000 genome Project variants database (http://www.1000genomes.or/); Esp6500siv2_all (http://evs.gs.washington.edu/EVS/), Inhouse databases (http://192.168.0.69/db/inhouse/); Exome Variant Server (version 0.0.30; http://evs.gs.washington.edu/EVS/); ExAC (http://exac. broadinstitute.org/); dbSNP (version 2.0; http://www.ncbi.nlm. nih.gov/projects/SNP/); and Clinvar (https://www.ncbi.nlm. nih.gov/clinvar/). The variants were selected according to the following five-step process, to select the potential pathogenic variants in the downstream analysis: i) Mutation reads $>10$ and mutation ratio $\geq 30 \%$; ii) following removal of the mutation from the search, the frequency showed more than $0.1 \%$ in the 1,000 Genomes, ESP6500, ExAC and Inhouse databases; iii) if the mutations existed in the In Normal database (MyGenostics), they were dropped; iv) removal of synonymous mutations or mutations in intronic region, since they were considered non-pathogenic; and v) after i), ii) and iii), if the mutations were in introns or were synonymous and reported in HGMD, they were included. All remaining variants were considered pathogenic. Finally, seven homozygous mutations were identified. Combined with the phenotype of the child, only the C12orf57 variant was confirmed as pathogenic. The C12orf57 pathogenic variant was screened using Sanger sequencing to amplify exon 1 (chr12-7053287) of C12orf57 (NM_138425). The segregation of the identified pathogenic variant was investigated in all the family members (Fig. 2A and B). The prediction of mutations was assessed using software, including Polyphen2_ HVAR_score, Polyphen2_HVAR_pred, PolyPhen_2_Predict and PolyPhen_2 (April 2010; http://genetics.bwh.harvard. edu/pph2/); MutationTaster, Mutation Taster_Predict (April 2009; http://www.mutationtaster.org/ChrPos.html); SIFT,SIFT_Predict (http://sift.jcvi.org/); LRT_score, LRT_pred (November 2009; http://www.genetics.wustl.edu/jflab/lrt_query. html); CADD_raw, CADD_phred (version 1.3; http://cadd. gs.washington.edu/).

Literature review. PubMed and Wanfang (http://www. wanfangdata.com.cn/index.html) databases were searched to retrieve studies using the keywords 'C12orf57', from inception to March 2019. The publication language was restricted to English and Chinese.

\section{Results}

Clinical manifestation. On admission, the patient was unable to hold his head at 6 months, and was unable to sit up straight at
10 months. Hospitalization had occurred three times because of severe pneumonia. Also, abnormal dysmorphic features (Figs. 1A and Video S1), such as frontal bossing, low-set ears, depressed nasal bridge, ocular hypertelorism, micrognathia and single transverse palmar crease were observed. Referring to the World Health Organization Anthro guidelines, the patient was $7 \mathrm{~kg}(\mathrm{Z}$-score=-2.17 SD) in weight and $65 \mathrm{~cm}$ $(Z$-score $=-2.75 \mathrm{SD})$ in length; the head circumference was $45 \mathrm{~cm}(\mathrm{Z}$-score $=0.02 \mathrm{SD})$.

An atrial septum defect $(4.5 \mathrm{~mm})$ was found by color Doppler ultrasonography (Fig. 1B). The magnetic resonance imaging (MRI) scans showed slightly expanded lateral ventricles and increased extra-axial spaces (Fig. 1C and D). The interictal electroencephalography showed sporadic spike-slow wave during waking. Any abnormalities of the eyes were not detected during the ophthalmological consultation. Furthermore, this patient was administered oxcarbazepine (8-15 $\mathrm{mg} / \mathrm{kg} /$ day) and anti-infection therapy (ceftazidime $100 \mathrm{mg} / \mathrm{kg} /$ day (intravenous infusion) for 5 days, followed by cefixime $5 \mathrm{mg} / \mathrm{kg} /$ day (oral administration) for 5 days. After 2 weeks, the patient was discharged and transferred to a local hospital for the recovery phase management.

During the 1-year follow-up, the patient was seizure-free for 1 month after the oxcarbazepine ( $26 \mathrm{mg} / \mathrm{kg} / \mathrm{day})$ was administered, and could only sit up straight for $<30 \mathrm{sec}$ at the age of 14 months. While there were still another six hospitalizations for pneumonia, only two had occurred in the previous 6 months and the patient had recovered easily. The atrial septum defect was not observed by color Doppler ultrasonography at the age of 19 months.

Genetic analysis. Exome sequencing revealed a homozygous pathogenic variant, c.3G $>C$ (p.Met1IIe), that was confirmed by Sanger sequencing. Furthermore, the pathogenic variant was segregated according to a strictly recessive model with full penetrance. The parents were tested as possible heterozygous carriers. A total of four heterozygous carriers of the C12orf57 pathogenic variant were detected; the patient's parents and their second degree relatives inherited the pathogenic variants from their grandparents (Fig. 2A and B).

C12orf57 encodes a protein that is evolutionarily conserved across representative species (Fig. 2C), according to HomoloGene (https://www.ncbi.nlm.nih.gov/homologene/). The actual in silico results are as follows: SIFT_score (0); SIFT_pred (D); Polyphen2_HDIV_score (0.072); Polyphen2_ HDIV_pred (B); Polyphen2_HVAR_score (0.008); Polyphen2_HVAR_pred (B); LRT_score (0); LRT_pred (D); CADD_raw (3.109); CADD_phred (22.5); Pathogenic variant Taster_score (1); Pathogenic variant Taster_pred (D). As a result, most of the in silico results predicted this biallelic missense pathogenic variant to be deleterious, wherein the pathogenic variant is localized at the start codon, abolishing the translation of C12orf57.

Literature review. Temtamy syndrome is not easy to distinguish clinically from other syndromes with similar phenotypes. As such, the present study only summarized cases with C12orf57 mutations. A total of 56 cases with $C 12 \operatorname{orf} 57$ pathogenic variants (Fig. S1) have been reported (3-7). All the cases were early onset, and the clinical features are summarized in Table I. 
A

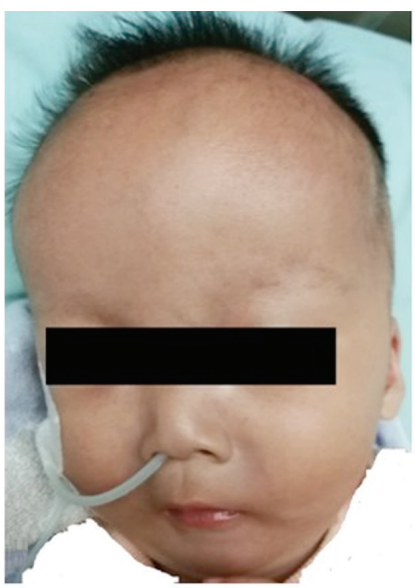

B

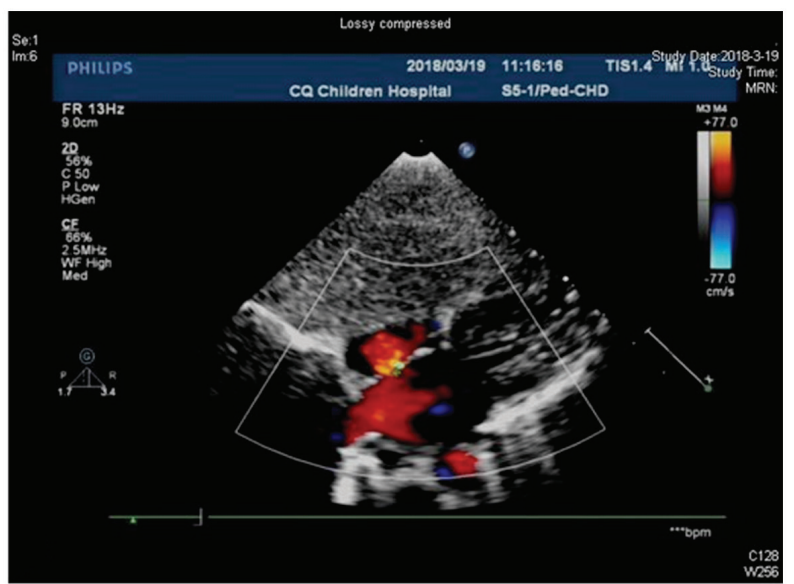

\section{Se:4}

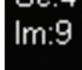

[HA]

Study Date: Study Time

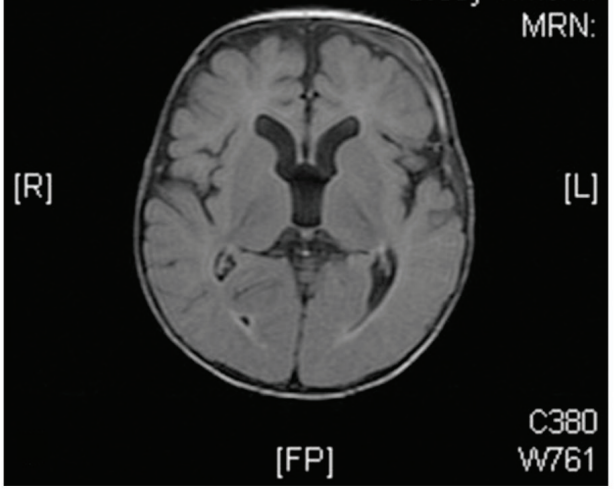

D $\frac{\substack{s, 2 \\ \text { lm. }}}{2}$

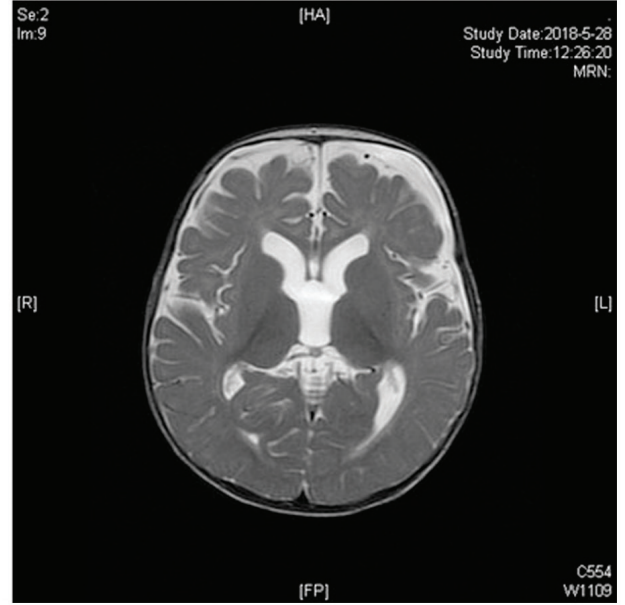

Figure 1. (A) Photograph of the patient (9 months), facial dysmorphism including frontal bossing, low set ears, depressed nasal bridge, ocular hypertelorism, and micrognathia. (B) The atrial septum defect (red arrow, $4.5 \mathrm{~mm}$ ) was detected through color Doppler ultrasonography. Magnetic resonance imaging (C) T1 and (D) T2 axial slices showed widened bilateral lateral ventricles and increased extracellular spaces.

A

B

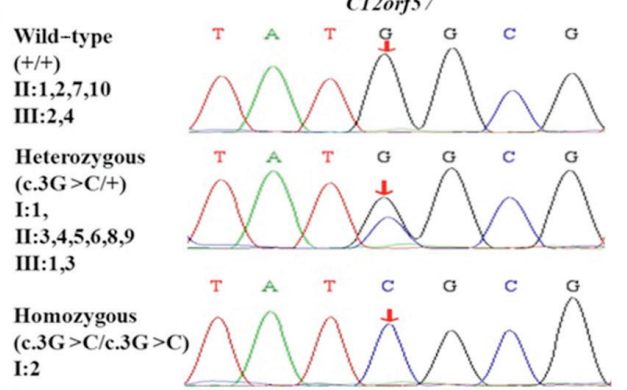

IV

III

II

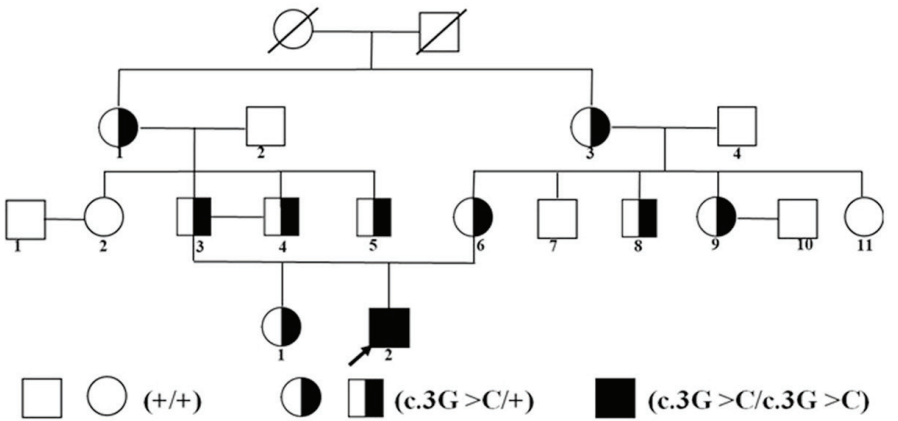

Homo sapiens/Human

Mus musculus:Mouse,

Canis lupus familisris (Dog)

Bos taurus/Bovine

Cavis porcellus/Guines pig)

Gallus gallus, Chicken

Takifugu rubripes

C

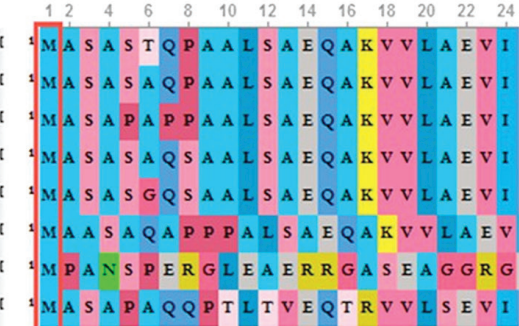

Figure 2. (A) Pedigree of the family. (B) The DNA sequencing showed that a novel C12orf57 pathogenic variant (c.3G>C, red arrows) co-segregates with the phenotype in the proband and the family members. (C) The C12orf57 missense pathogenic variant, as shown by the red arrow in (B) is localized in a highly conserved amino acid sequence among representative species. 
Table I. Chromosome 12 open reading frame 57 pathogenic variants and phenotypes of all the affected cases from the literature and the current report.

\begin{tabular}{|c|c|c|c|}
\hline Characteristic & The present case & Literature $(\mathrm{n}=56)$ & Total, \% \\
\hline \multicolumn{4}{|l|}{ Basic characteristics } \\
\hline Early onset & 1 & 56 & 100 \\
\hline Consanguineous & 1 & 49 & 87.7 \\
\hline \multicolumn{4}{|l|}{ Ethnic origin } \\
\hline Saudi Arabia & & 25 & 43.9 \\
\hline Kuwait & & 9 & 15.8 \\
\hline United Arab Emirates & & 9 & 15.8 \\
\hline Libya & & 4 & 7.0 \\
\hline Palestine & & 4 & 7.0 \\
\hline Oman & & 3 & 5.3 \\
\hline Germany & & 2 & 3.5 \\
\hline China & 1 & & 1.8 \\
\hline \multicolumn{4}{|l|}{ Genetics } \\
\hline c.1A>G,p.(Met1?) & & 45 & 78.9 \\
\hline c. $3 \mathrm{G}>\mathrm{C}, \mathrm{p} .($ Met1I) & 1 & & 1.8 \\
\hline c. $53-2 \mathrm{~A}>\mathrm{G}$ & & 3 & 5.3 \\
\hline c. $184 \mathrm{C}>\mathrm{T}, \mathrm{p} .\left(\mathrm{Gln} 62^{*}\right)$ & & 2 & 3.5 \\
\hline c.-3_2delinsG & & 2 & 3.5 \\
\hline c. $43 \mathrm{C}>\mathrm{T}, \mathrm{p} .\left(\mathrm{G} \ln 15^{*}\right)$ & & 2 & 3.5 \\
\hline c. $229+2 \mathrm{~T}>\mathrm{C}$ & & 1 & 1.8 \\
\hline c. $152 \mathrm{~T}>\mathrm{A}, \mathrm{p} .(\mathrm{Leu} 51 \mathrm{Gln})$ & & 1 & 1.8 \\
\hline
\end{tabular}

Phenotype

Neurological findings

Developmental delay

Seizures

$56 / 56$

100

Absent speech

$41 / 56$

73.7

Generalized hypotonia

$41 / 55$

74.5

Delayed speech

$40 / 56$

71.9

Autistic behavior

$20 / 55$

37.5

Spasticity

$40 / 55$

72.7

20/56

35.1

Dysmorphic features

Dysmorphic faces

$36 / 55$

66.1

Microphthalmia

9/55

14.3

Ophthalmology

Ocular anomalies

$26 / 55$

Coloboma

Congenital heart disease

Atria septal defect

Ventricular septal defect

Pulmonic stenosis

Brain imaging

Abnormal corpus callosum

Abnormal thalamic size

20/51

Abnormal septum pellucidum

$19 / 50$

Abnormal white matter

$19 / 50$

Abnormal anterior commissure

$11 / 51$

21.2

Ventriculomegaly

$17 / 50$

35.3

Others

Recurrent pneumonia

N/A 


\section{Discussion}

Temtamy syndrome is an extremely rare disorder, and only a limited number of studies have been conducted (1-7). The present study reports the first East Asian patient with a novel C12orf57 homozygous pathogenic variant; all other heterozygous carriers in the family were clinically healthy. The inheritance pattern of this pedigree was in accordance with autosomal recessive inheritance with complete penetrance.

Pathogenic variants in C12orf57 were first reported to cause Temtamy syndrome in 2013 (3-5), and the affected individuals were recently described to have variable phenotypes beyond this syndrome (5). The c. $1 \mathrm{~A}>\mathrm{G}$ pathogenic variant in the C12orf57 start codon has been reported as the most frequent pathogenic variant $(45 / 56,80.3 \%)$. Interestingly, the pathogenic variant in this start codon can severely reduce the protein levels, suggesting a loss of function as the mechanism of action behind the disease. Moreover, the pathogenic variant (c. $3 \mathrm{G}>\mathrm{C})$ in the current case was located at the same start codon of C12orf57, but at different bases. This suggests that the $c .3 \mathrm{G}>\mathrm{C}$ pathogenic variant may affect the protein expression level and may lead to the occurrence of disease. Bioinformatics analysis indicated that the aforementioned pathogenic variant is the most likely mechanism of action behind the pathogenesis. Since the parents of the patient are first-cousin consanguineous relatives, whether the patient had other additional homozygous pathogenic variants was investigated, but no positive results were obtained.

All the patients with C12orf57 pathogenic variants identified in the literature review exhibited global developmental delay in concurrence with hypotonia. Compared to the previously reported cases of epilepsy (73.2\%), relatively refractory (37.5\%) and low frequency of seizure-free (15.6\%), as found in the literature review, the seizures in the current case were controlled. No recurrence of seizures occurred after the patient was medicated with oxcarbazepine. Although the dysmorphic features are not distinctly recognized among patients with C12orf57 pathogenic variants, the current case had many similar dysmorphic facial features (65.5\%), including frontal bossing, low set and posteriorly rotated ears, depressed nasal bridge, hypertelorism, micrognathia, up-slanted palpebral fissures and microphthalmia, but without epicanthal folds. Previously reported abnormalities of the eyes (such as chorioretinal coloboma), iris or optic nerve were not found in this present case. C12orf57 was once reported to cause colobomatous microphthalmia, which seems to be invariably associated with profound global developmental delay, seizures and defects of the corpus callosum, but these cases were later summarized to be Temtamy syndrome by the same research group $(4,5)$. This present study identified colobomatous microphthalmia to be a clear sign of this syndrome.

Abnormalities of the corpus callosum were included in the classical characteristics of this disease and may be observed in approximately two-thirds of patients with C12orf57 pathogenic variants. However, no similar corpus callosum changes were demonstrated and MRI scanning of the brain revealed only slight cerebral dysplasia in the present case. Reportedly, atrial septum defects are the most frequent congenital heart defect, which was also observed in the present case, at $4.5 \mathrm{~mm}$.
Additionally, the present patient was particularly susceptible to infection, developing severe pneumonia every 2-3 months leading to hospitalization for several weeks. No similar recurrent pneumonia has been mentioned in the previous studies. During the follow-up, the recurrent infections were less frequent in the latter 6 months; therefore, it is considered that the recurrent infections may be partly associated with the atrial spectrum defects, which was not detected at previous visits. Therefore, additional cases could substantiate the findings if recurrent infection is a feature of patients with $C 12$ orf57 pathogenic variants. Currently, the patient is 20 months old and no more new outcomes have been observed. Follow-up observations are needed to confirm the presence of further phenotypes.

The present study has some limitations. Detailed information on reported cases of pathogenic C12orf57 pathogenic variants is limited (for example, familial aggregation, life expectancy and type of seizures). Furthermore, the phenotype-genotype correlation cannot be confirmed since the majority $(45 / 57)$ of the reported cases had the same pathogenic variant, c. $1 \mathrm{~A}>\mathrm{G}$, p.(Met1?), and the other different genetic variants were only observed in one or two cases.

In conclusion, a novel homozygous C12orf57 pathogenic variant (c. $3 \mathrm{G}>\mathrm{C})$ was identified in a patient with developmental delay, epilepsy and dysmorphic facial appearance. The C12orf57 pathogenic variant site has not been published previously, and this was the first reported East Asian case with Temtamy syndrome to the best of our knowledge. The present results expanded the spectrum of C12orf57 pathogenic variants, as well as the ethnic backgrounds of the affected cases.

\section{Acknowledgements}

Not applicable.

\section{Funding}

This study was partially supported by a grant obtained from the Children's Hospital of Chongqing Medical University (grant no. HJYN2013-4).

\section{Availability of data and materials}

The datasets used and/or analyzed during the current study are available from the corresponding author on reasonable request.

\section{Authors' contributions}

YW, YL and ML carried out the diagnosis and treatment of the case, collected the data, and performed literature review. MZ designed and supervised the study. XZ, SL, LJ and XL were involved in the management of the patient and approved the final version of the manuscript.

\section{Ethics approval and consent to participate}

The ethics approval for this study was obtained through the Institutional Review Board of Children's Hospital of Chongqing Medical University (Chongqing, China; no. 2018-64). 


\section{Patient consent for publication}

The parents provided consent for the case report and for images of the patient's face to be published for educational purposes.

\section{Competing interests}

The authors declare that they have no competing interests.

\section{References}

1. Temtamy SA, Salam MA, Aboul-Ezz EH, Hussein HA, Helmy SA and Shalash BA: New autosomal recessive multiple congenital abnormalities/mental retardation syndrome with craniofacial dysmorphism absent corpus callosum, iris colobomas and connective tissue dysplasia. Clin Dysmorphol 5: 231-240, 1996.

2. Temtamy SA, Abdel Salam M, Aboul-Ezz EH, Hussein HA, Helmy SMH and Shalash BA: A new autosomal recessive MCA/MR syndrome with craniofacial dysmorphism, absent corpus callosum, iris colobomas and connective tissue dysplasia. (Abstract) Am J Hum Genet 49 (Suppl): S167, 1991.
3. Akizu N, Shembesh NM, Ben-Omran T, Bastaki L, Al-Tawari A, Zaki MS, Koul R, Spencer E, Rosti RO, Scott E et al: Whole-exome sequencing identifies mutated c12orf57 in recessive corpus callosum hypoplasia. Am J Hum Genet 92: 392-400, 2013.

4. Zahrani F, Aldahmesh MA, Alshammari MJ, Al-Hazzaa SA and Alkuraya FS: Mutations in c12orf57 cause a syndromic form of colobomatous microphthalmia. Am J Hum Genet 92: 387-391, 2013.

5. Alrakaf L, Al-Owain MA, Busehail M, Alotaibi MA, Monies D, Aldhalaan HM, Alhashem A, Al-Hassnan ZN, Rahbeeni ZA, Murshedi FA, et al: Further delineation of temtamy syndrome of corpus callosum and ocular abnormalities. Am J Med Genet A 176: 715-721, 2018.

6. Platzer K, Huning I, Obieglo C, Schwarzmayr T, Gabriel R, Strom TM, Gillessen-Kaesbach G and Kaiser FJ: Exome sequencing identifies compound heterozygous mutations in C12orf57 in two siblings with severe intellectual disability, hypoplasia of the corpus callosum, chorioretinal coloboma, and intractable seizures. Am J Med Genet A 164A: 1976-1980, 2014.

7. Salih MA, Tzschach A, Oystreck DT, Hassan HH, AlDrees A, Elmalik SA, El Khashab HY, Wienker TF, Abu-Amero KK and Bosley TM: A newly recognized autosomal recessive syndrome affecting neurologic function and vision. Am J Med Genet A 161A: 1207-1213, 2013. 